9 Agara AJ, Makanjoula AB. Pattern and pathway of psychiatric presentation at the out-patient clinic of a neuropsychiatric hospital in Nigeria. Niger J Psychiatry 2006; 4: 30-4.

10 Pieters G, Speybrouck E, De Gucht V, Joos S. Assaults by patients on psychiatric trainees: frequency and training issues. Psychiatr Bull 2005; 29: $168-70$.

11 Fottrell E. A study of violent behaviour among patients in psychiatric hospitals. Br J Psychiatry 1980; 136: 216-21.
12 Owen C, Tarantello C, Jones M, Tennant C. Violence and aggression in psychiatric units. Psychiatr Serv 1998; 49: 1452-7.

13 Haller RM, Deluty RH. Assaults on staff by psychiatric in-patients. A critical review. Br J Psychiatry 1988; 152: 174-9.

14 Caldwell MF. Incidence of PTSD among staff victims of patient violence. Hosp Community Psychiatry 1992; 43: 838-9.

\title{
Improving physical health monitoring in secondary care for patients on clozapine
}

\author{
Philippa J. Bolton ${ }^{1}$
}

The Psychiatrist (2011), 35, 49-55, doi: 10.1192/pb.bp.109.028753

${ }^{1}$ County Durham and Darlington NHS Foundation Trust

Correspondence to Philippa J. Bolton (philippa.bolton@ntw.nhs.uk)

\begin{abstract}
Aims and method To assess how well psychiatric teams follow up abnormal results from physical health monitoring of patients in a secondary care clozapine clinic compared with follow-up in a specialist secondary care physical health clinic, using an audit/re-audit method of comparison. Prevalence data for cardiovascular risk factors within the clinic population were also obtained.
\end{abstract}

Results Substantial and statistically significant $(P<0.01)$ improvements in follow-up of abnormal results were made following the introduction of a specialist clinic compared with treatment as usual. Prevalence of all cardiovascular risk factors among the patients on clozapine was very high compared with the general population.

Clinical implications Patients on clozapine have significant and multiple cardiovascular risk factors, which may be best managed within a specialist secondary care physical health clinic that can follow up abnormal results and coordinate care across primary and secondary care.

Declaration of interest None.
It has been recognised for some years that people with a diagnosis of schizophrenia have notoriously poor access to healthcare. Life expectancy is reduced by $20 \%$, with $60 \%$ of the excess mortality due to physical illness. ${ }^{1}$ The large-scale US Clinical Antipsychotic Trials in Intervention Effectiveness (CATIE) study identified major deficiencies in lack of diagnosis and treatment for cardiovascular risk factors in this population. ${ }^{2}$ The 2006 guidelines for schizophrenia from the National Institute for Health and Clinical Excellence (NICE) recognised these problems and identified the need for these patients to receive appropriate physical healthcare. ${ }^{3}$ The NICE guidelines stipulate that the majority of antipsychotic monitoring should take place in primary care, but that patients who cannot access a general practitioner (GP) should receive care from secondary care psychiatric services. The guidelines also stipulate that psychiatric case notes should document who is taking responsibility for care. Furthermore, it follows that if monitoring takes place in primary care, then results need to be disseminated to psychiatrists in secondary care to ensure that they can adequately weigh up the risk-benefit ratio of prescribing antipsychotics.

Three years on, the question is how well have these guidelines been implemented?

The largest British study ${ }^{4}$ pertinent to this question to date was conducted in 2007 - a year after the NICE guidelines were released. This study assessed the prevalence of untreated cardiovascular risk factors out of a cohort of 1966 patients in assertive outreach teams. Screening rates were low; for every one person with diabetes, one was missed; for dyslipidaemia, seven were missed; and for hypertension, four were missed. Questionnaires sent to teams showed that a third of staff did not think they were responsible for physical healthcare, half did not know how to interpret results, and many teams had no basic equipment to do health checks. This suggests that further work needs to be done in secondary care teams to ensure better physical healthcare. 
The aim of this study was to assess the quality of an important aspect of physical healthcare provision, which is to evaluate how well abnormal blood results are followed up within secondary care services. To do this a group of patients who received clozapine were selected. Clozapine is an antipsychotic with evidence of an association with multiple cardiovascular risk factors - one piece of evidence estimates that clozapine saves around 492 schizophrenic patients from suicide per 100000 clozapine patient-years, but weight gain associated with the drug kills 416 patients. ${ }^{5}$ Patients have their full blood count monitored frequently because of the risk of agranulocytosis. Most of this monitoring is done in secondary care clinics, often in conjunction with liver function tests, electrocardiogram (ECG), lipid tests, glucose tests, and measurement of weight, blood pressure and pulse. ${ }^{6}$

This study proposes a service design to evaluate and improve cardiovascular risk profiles in this population. Data for cardiovascular risk factors in the clinic cohort were obtained that can inform psychiatrists in the UK of the severity of the problems they are likely to face.

\section{Method}

\section{Description of original service}

Patients on clozapine at the clinic at the County Hospital in Durham attend for regular full blood count monitoring, as stipulated by Zaponex, the company that supplies the drug, owing to the risk of agranulocytosis. Patients attend weekly for the first 18 weeks, then every 2 weeks up to 1 year, and then monthly. Monitoring is also carried out for lipids, fasting glucose, liver function, blood pressure, pulse and weight. In 2006 the monitoring of these parameters was as follows:

- blood pressure, glycated haemoglobin $\left(\mathrm{HBA}_{1 \mathrm{c}}\right)$, weight monitored every 3 months

- liver function tests, urea and electrolytes - monitored every 6 months

- fasting lipid profile, thyroid function tests - monitored yearly.

Monitoring was carried out by nursing staff, with follow-up from consultant teams. The team consisted of two qualified mental health nurses, who between them provided ten sessions for the clinic, and a healthcare assistant who provided six sessions for the clinic. A doctor provided one session for any medical queries that arose. A team manager from a community mental health team was in place; the manager provided no clinical sessions but gave supervision for nursing staff. No standards or procedures for follow-up existed. An audit was done to assess the proportion of abnormal results that were followed up correctly compared with the audit standard of a 100\% correct follow-up rate. Correct follow-up was defined by guidelines that were taken from national guidelines, literature reviews or studies ${ }^{6-13}$ and advice from experts in the relevant fields. The guidelines (see an online supplement to this paper) aim to ensure that patients get the correct follow-up by ensuring that appropriate blood tests and other investigations are done at the appropriate time and then referred, as required, to the correct service.

\section{The intervention}

Following the baseline survey, a new service model was designed. The whole team was involved in this, and monthly team meetings were held for service development, with supervision occurring for the nurses approximately once a week from the doctor. The new service model consisted of all the results obtained being followed up by the doctor within the clinic using the guidelines, with information regarding abnormal results going to the GP and care coordinator via a letter. Nursing staff carried out the appropriate tests and gave explanations to the patients. The aims of establishing correct follow-up were threefold: first, to ensure that patients received appropriate and timely blood tests to identify any pathology at an early stage; second, to establish that clozapine was not the direct cause of the abnormality (and thus needed to be withdrawn); and third, to ensure that patients were referred appropriately for further management, or clarification of diagnosis, to primary or secondary care. The guidelines were used to provide an evidence-based system of triage. For example, if a patient presents asymptomatically with a blood pressure reading over $140 / 90 \mathrm{mmHg}$ but under $180 / 110 \mathrm{mmHg}$, the blood pressure is measured again at the end of the appointment and again at the next two clozapine appointments, which are 4 weeks apart. If the blood pressure continues to be above $140 / 90 \mathrm{mmHg}$, then hypertension can be diagnosed and the patient is referred to their GP for follow-up, which may involve the GP prescribing antihypertensive medication. Under such a system, only a small minority of patients would need GP follow-up; resources could be concentrated on those patients, to ensure that follow-up is complied with. Furthermore, psychiatrists could make confident decisions about the risks of patients remaining on clozapine.

Nursing staff ensured the letters to GPs were acted upon by contacting the patients, GPs, care coordinators and, if relevant, primary carers or support staff, to make sure the patients had attended the GP and that a report from the GP was obtained. General practitioners have access to all blood results via electronic access to the same laboratories that are used by the clinic. Any medication or follow-up was then put in the patient's care plan by the nursing team. When necessary, the healthcare assistant accompanied patients to GP surgeries and, for patients with very complex needs, met with the GPs for case reviews. The nursing staff spent time explaining the information to the patient and working with the patient to improve the problem, e.g. offering to link the patient to the smoking cessation network if they had hypertension. Annual consultations with patients conducted jointly by the doctor and nursing staff to discuss physical health issues were started, along with cardiovascular risk profile letters, as per recommendations in the literature. ${ }^{7}$ These included intervention plans for both primary and secondary care notes. A flow chart summarising the intervention is shown in Fig. 1. 


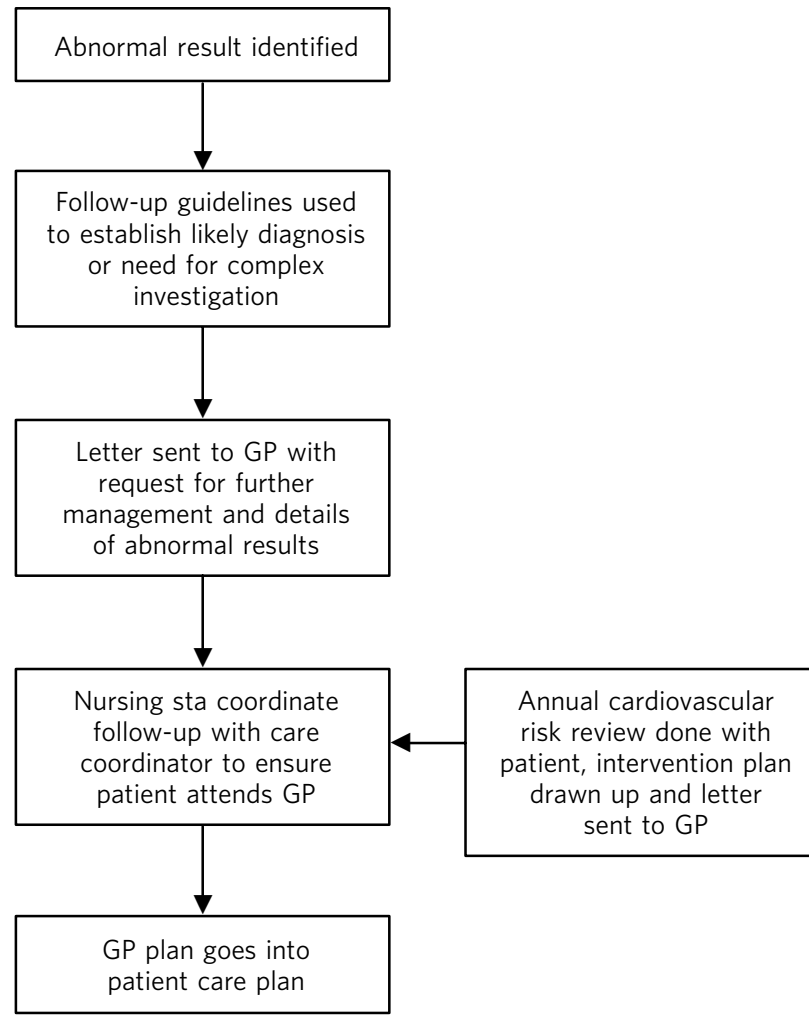

Fig 1 Flow chart to demonstrate the intervention process. GP, general practitioner.

\section{Changes to monitoring requirements}

Some of the monitoring requirements for the 2007-08 cohort were changed, based on updates from guidelines and the literature. ${ }^{6}$ In the first year:

- fasting lipids and glucose and blood pressure were measured every 3 weeks

- weight was measured weekly for the first 3 months and monthly thereafter

- liver function tests and urea and electrolytes were measured every 6 months.

This reflects the increased risk of developing problems in the first year compared with subsequent years.

After the first year:

- blood pressure and weight were monitored every 3 weeks

- liver function tests, urea and electrolytes and fasting glucose were monitored every 6 months

- the fasting lipid profile was monitored yearly.

To create an annual cardiovascular risk profile, patients were assessed for smoking status, age, gender, body mass index (BMI), 10-year cardiovascular risk (Framingham criteria; http://cvrisk.mvm.ed.ac.uk) for patients aged over 35 years, presence of metabolic syndrome, and family history of diabetes and cardiovascular disease.

Follow-up was audited and the baseline data were compared with the data collected after the intervention was in place, to assess how effective the intervention had been in improving follow-up of abnormal results.

\section{Statistical analysis}

Statistical analysis was used to compare the follow-up of abnormal results in 2006 with those from 2007. Fisher's exact test was used to determine whether the effect of the intervention was significant; this test was used as the data were nominal and the number of observations in some of the cells was fewer than five. $P$-values were found for each variable; $P<0.05$ was deemed significant in establishing the effectiveness of the intervention. Analysis was done using StatsDirect version 2.6.6 for Windows (available from www.statsdirect.com). Odds ratios were also calculated.

\section{Results}

\section{Participants}

A total of 56 patients attended the clozapine clinic at Durham County Hospital in 2006. Six patients were not included in the audit as they were either in-patients or lived in nursing homes at the time and the notes were not accessible. One person left the clinic and so was not included, as not all relevant details were obtained. This gave a total of 49 patients in the 2006 cohort.

In the year between assessments, one person stopped taking clozapine because of hereditary cardiomyopathy being discovered, two patients discontinued the drug against medical advice, and two moved out of the area.

Of the original cohort, 44 patients remained in 2007. Seven new patients were added to the clinic - four started on clozapine in that interval, two moved into the area having already been established on clozapine, and one of the previous in-patients came back to the clozapine out-patient clinic. This gave a total of 51 patients in the 2007 cohort (Fig. 2).

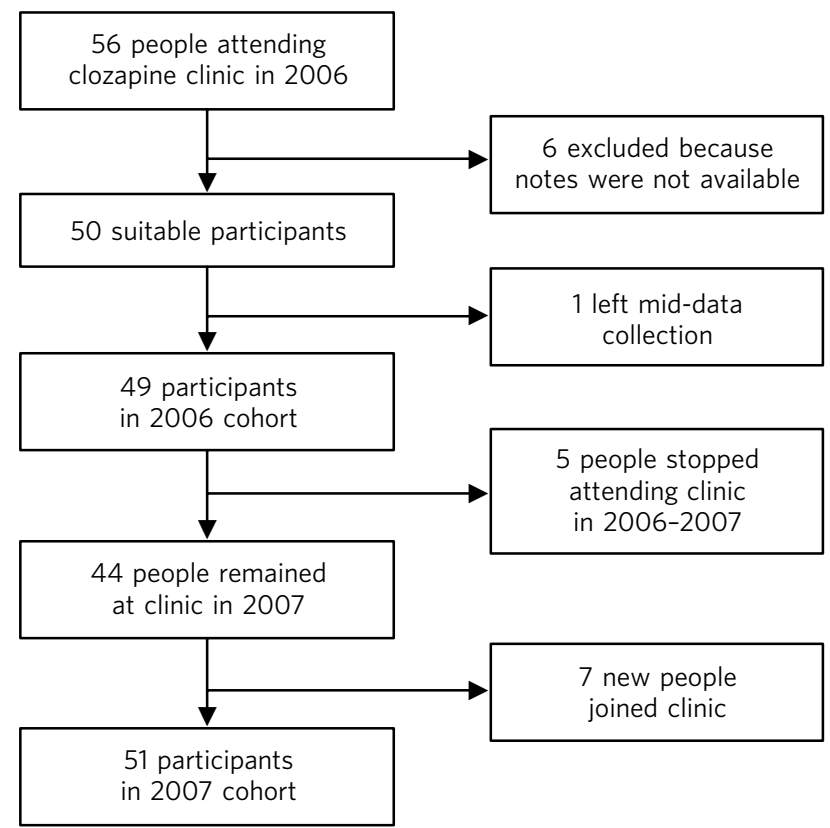

Fig 2 Flow chart of participants through the study. 


\section{Demographic comparison between 2006 and 2007}

The patients had a mean age of 40 years (38.5 years in 2006 , 40.2 years in 2007) and were mostly male (70\% in 2006 , $75 \%$ in 2007). About two-thirds of the sample population were smokers (69\% in 2006, 67\% in 2007). Almost all patients were overweight or obese (three patients in 2006 and one in 2007 had a normal body mass index, BMI): approximately a third were overweight (39\% in 2006, 37\% in 2007) and just under two-thirds were classed as obese (19.5\% in $2006,27 \%$ in 2007), severely obese (19.5\% in 2006 , $22 \%$ in 2007 ) or morbidly obese ( $14 \%$ in $2006,12 \%$ in 2007 ). The mean length of time on clozapine was 5 years in 2006 and 7.2 years in 2007 .

All patients were White British, except for one patient of mixed ethnicity. This ethnic mix is broadly representative of the general population within Durham and was the same in both cohorts (Table 1).

\section{Follow-up of abnormal results}

Table 2 shows the number of patients each year, how many had abnormal or out-of-date results, and how many of those results were incorrectly followed up.

Out-of-date routine monitoring was included in both the abnormal results and the incorrectly investigated results, because if routine monitoring is not done correctly then it must be assumed, until proven otherwise, that the patient may be at risk.

\section{Blood glucose}

In 2006, glycated haemoglobin $\left(\mathrm{HBA}_{1 \mathrm{c}}\right)$ was used to measure blood glucose every 6 months. Sixteen patients (32\%) had an abnormal result, of whom five were known to have pre-existing diabetes. Of these 16 patients, $12(75 \%)$ were incorrectly followed up. In 2007, the monitoring was changed to fasting blood glucose or $\mathrm{HBA}_{\mathrm{lc}}$ for those who would not fast (eight participants). Thirteen (25\%) abnormal or out-of-date results were recorded; 8 of these 13 patients had diabetes. Of these 13 patients, $4(30 \%)$ were incorrectly followed up. The improvement seen in follow-up

\begin{tabular}{|c|c|c|}
\hline \multicolumn{3}{|c|}{$\begin{array}{l}\text { Table } 1 \text { Summary of demographic information for } 2006 \\
\text { and } 2007 \text { cohorts }\end{array}$} \\
\hline & $\begin{array}{c}2006 \\
\text { cohort }^{\text {a }}\end{array}$ & $\begin{array}{c}2007 \\
\text { cohort }^{b}\end{array}$ \\
\hline Mean age, years & 38.5 & 40.2 \\
\hline Males, $n(\%)$ & $38(70)$ & $38(75)$ \\
\hline Females, $n(\%)$ & $15(30)$ & $13(25)$ \\
\hline Non-smokers, $n(\%)$ & $15(31)$ & $17(33)$ \\
\hline Smokers, n (\%) & $34(69)$ & $34(67)$ \\
\hline Morbidly obese (BMI >40), n (\%) & $7(14)$ & $6(12)$ \\
\hline Severely obese (BMI 35-39.9), n (\%) & $10(20)$ & $11(22)$ \\
\hline Obese (BMI 30-34.9), n (\%) & $10(20)$ & $14(27)$ \\
\hline Overweight (BMI 25-29.9), n (\%) & $20(39)$ & $19(37)$ \\
\hline Normal weight (BMI 20-24.9), n (\%) & $3(6)$ & $1(2)$ \\
\hline Unknown, $n(\%)$ & $1(2)$ & $0(0)$ \\
\hline $\begin{array}{l}\text { Mean length of time on clozapine, } \\
\text { years }\end{array}$ & 5 & 7.2 \\
\hline Patients in first year of treatment, $n$ & 7 & 4 \\
\hline
\end{tabular}

BMI, body mass index.

a. Total number of patients 53 .

b. Total number of patients 51 .

from 2006 to 2007 was deemed statistically significant $(P=0.0121$, odds ratio $\mathrm{OR}=8.25,95 \% \mathrm{CI} 1.3-56.2)$.

\section{Blood pressure}

In 2006, 17 patients (35\%) had abnormal blood pressure readings, of whom $16(94 \%)$ were incorrectly followed up. In 2007 , a total of $16(31 \%)$ abnormal or out-of-date readings were recorded, of which only $6(37.5 \%)$ were incorrectly followed up. The difference in results between the years was deemed statistically significant $(P=0.0008, \mathrm{OR}=26.7,95 \%$ CI 2.5-1226.9).

\section{Dyslipidaemia}

In 2006, 37 patients (75\%) either had abnormal results or had never had blood lipids measured. Of these 37 patients,

Table 2 Patients with abnormal results for each year and how well they were followed up

\begin{tabular}{|c|c|c|c|c|}
\hline \multirow[b]{2}{*}{ Test } & \multicolumn{2}{|c|}{ Patients with abnormal results } & \multicolumn{2}{|c|}{$\begin{array}{l}\text { Patients with abnormal results followed up in } \\
\text { accordance with guidelines }\end{array}$} \\
\hline & $2006(N=49)$ & $2007(N=51)$ & $2006(N=49)$ & $2007(N=51)$ \\
\hline $\mathrm{HBA}_{1 c}$ or fasting glucose & $\begin{array}{l}5 \text { with diabetes } \\
11 \text { abnormal } \\
\text { Total: } 16(32 \%)\end{array}$ & $\begin{array}{l}8 \text { with diabetes } \\
2 \text { abnormal } \\
3 \text { out of date } \\
\text { Total: } 13(25 \%)\end{array}$ & $12(75 \%)$ & $4(30 \%)$ \\
\hline Blood pressure & $17(35 \%)$ & $\begin{array}{l}15 \text { abnormal } \\
1 \text { out of date } \\
\text { Total: } 16(31 \%)\end{array}$ & $16(94 \%)$ & $6(38 \%)$ \\
\hline Dyslipidaemia & $\begin{array}{l}15 \text { abnormal } \\
22 \text { not done } \\
\text { Total: } 37(75 \%)\end{array}$ & $\begin{array}{l}22 \text { high cholesterol } \\
26 \text { high triglycerides } \\
32 \text { one or both } \\
8 \text { out of date } \\
\text { Total: } 40(78 \%)\end{array}$ & $37(100 \%)$ & $14(35 \%)$ \\
\hline Liver function tests & $\begin{array}{l}15 \text { abnormal } \\
1 \text { out of date } \\
\text { Total: } 16(32 \%)\end{array}$ & $\begin{array}{l}17 \text { abnormal } \\
2 \text { out of date } \\
\text { Total: } 19(37 \%)\end{array}$ & $16(100 \%)$ & $2(14 \%)$ \\
\hline
\end{tabular}


none had correct follow-up. In 2007, 22 patients had high cholesterol, 26 had high triglycerides and 32 had one or both abnormalities. Results for eight patients were out of date because they had not fasted. This gave a total of 40 patients $(78 \%)$. Of these 40 patients, 14 (35\%) were incorrectly followed up, an improvement of $65 \%$ on the previous year. The improvement in the results was deemed statistically significant $(P<0.0001)$. As there were no correct follow-ups in the 2006 results, $\mathrm{OR}=\infty$. The lowerlevel confidence interval is 14.1, and this gives the minimum odds ratio from these results.

\section{Liver function tests}

In 2006, 16 patients (32\%) had abnormal liver function tests, with one of these being out of date. Of these 16, none was followed up correctly. In comparison, data from 2007 demonstrate that a similar proportion of patients $(n=17$, $37 \%$ ) had abnormal results, but of these only 2 patients (14\%) were incorrectly followed up. The improvement was deemed statistically significant $(P<0.0001)$.

There were no correct follow-ups in the 2006 results, so $\mathrm{OR}=\infty$, but 14.7 is the lower-level confidence interval, which gives the minimum odds ratio from these results.

The results and analyses reveal that the effect of the intervention across all variables was statistically significant. Follow-up was significantly improved for all blood results, with the effect being most evident in lipid and liver function test follow-up $(P<0.0001)$, particularly as no patients were followed up correctly in the original group. Blood pressure follow-up was also significantly improved $(P<0.0008)$, and a less marked, but still significant, effect for glucose followup $(P<0.01)$ was also seen.

\section{Prevalence data for cardiovascular risk factors}

Table 3 details the point prevalence of risk factors relating to the development of future cardiovascular disease in this cohort of 50 patients. Prevalences of all the risk factors for this group are higher than would be expected in a general adult population, with a mean age of 40 years. Ten patients (20\%) had hypertension, 11 had diabetes (22\%), and 3 had impaired fasting glycaemia (6\%), which is a risk factor for developing diabetes. Over half the cohort had abnormal lipids, either high cholesterol $(42 \%)$ or high triglycerides $(50 \%)$, or both. Obesity was prevalent in 31 patients $(62 \%)$,

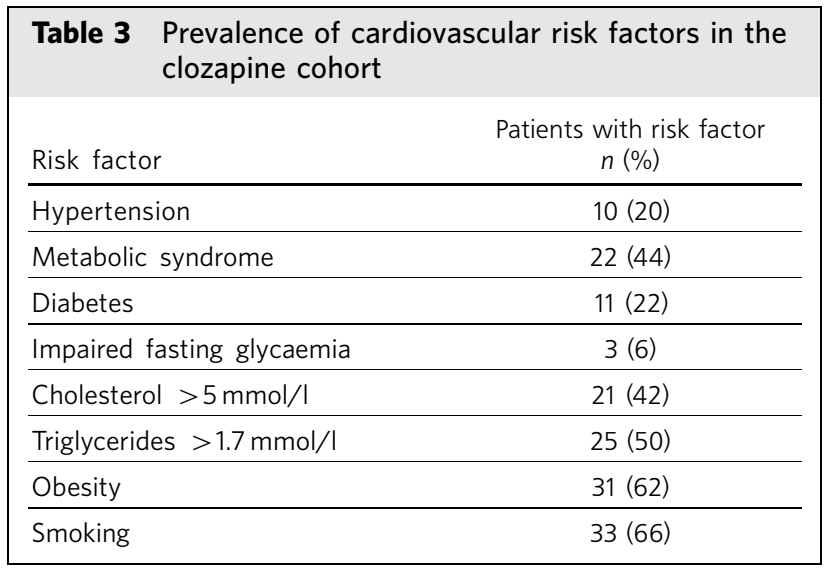

and 33 patients (66\%) were smokers. For patients aged over 35 years, a 10-year cardiovascular risk calculation was done. There were 32 patients over the age of 35 years, of whom 10 had a cardiovascular risk of $10-20 \%$ (medium risk) and 9 had a cardiovascular risk over 20\% (high risk). Of 43 patients who were able to provide information on their family history, 10 had a positive family history of cardiovascular disease.

Of the patients in this part of the study, three were also taking aripiprazole, one was on lithium, six were on valproate, one was on risperidone, one was on haloperidol, one was on amisulpride and one was on sulpiride. In total, 14 patients took other antipsychotics or mood stabilisers.

\section{Discussion}

The results indicate that monitoring and follow-up in a specialist clinic showed significantly better results than those seen with follow-up by separate consultant teams. All statistical tests showed $P<0.01$; the ORs and $95 \%$ CIs were all in favour of the new method for monitoring, although the confidence intervals were wide, which is a reflection of the small sample size.

The prevalence data on patients at the clozapine clinic after the intervention generally reflect that found in the literature. The quantity of literature relating to clozapine patients is small and there are no UK studies. In this study, $22 \%$ of patients had diabetes or impaired fasting glycaemia, which is lower than the $34 \%$ found in Henderson et al's 5 -year follow-up study ${ }^{14}$ but similar to the $27 \%$ quoted in more recent publications. ${ }^{15}$ Hypertension was found to have a $20 \%$ prevalence compared with $27 \%$ in a 2004 study. ${ }^{16}$ However, dyslipidaemia was found to be very high in the present study, at $88 \%$. There are no studies that specifically look at dyslipidaemia in the clozapine population, but in patients taking atypical antipsychotics it is estimated to present in at least $50 \%$ of patients, with recognition that this figure is higher in those taking clozapine. Metabolic syndrome in this study was found to be $44 \%$, compared with $53 \%$ in the literature. ${ }^{17}$ The slightly lower prevalence of cardiovascular risk factors may reflect differences between the US and UK populations; or, as all studies are fairly small, it may simply reflect normal variance in sample populations and a true estimate may be found by meta-analysis if more small-scale studies were to be done. In summary, the high incidence of cardiovascular risk factors found in this study supports the need for more investment in services for this population.

\section{Methodological considerations}

The study design was an audit/re-audit cycle of the effect of the intervention in the clozapine clinic population, comparing quality of follow-up pre- and post-intervention. A randomised controlled trial would have been a goldstandard design to test the effectiveness of an intervention; however, this would have required access to a much larger population in order to obtain a random sample. Patients in both groups have fairly similar demographic information, which is unsurprising, as 44 of the patients were the same in both cohorts. The main significant difference is the number 
of patients in the first year of treatment, with seven in 2006 and only four in $2007 ; 86 \%$ of patients were the same between cohorts. The $14 \%$ of patients who did not remain the same between the 2 years would have been unlikely to affect results significantly. Patients can alter outcomes only by refusing tests or not fasting, which would affect only lipid results because random glucose or glycated haemoglobin $\left(\mathrm{HBA}_{\mathrm{lc}}\right)$ can be used as an alternative to fasting tests. Only one patient routinely refused blood tests, and he remained constant within both groups. Fasting could have altered results slightly, with the slightly different cohort, but in reality very few patients did not fast (8 of 51 in the second year), and so any effect would have been minor. The other factor is the first-year variable. The most risky time for abnormal results is the first year, hence the increase in monitoring. In the second year, the patients are new to the cohort but had they been excluded, the first-year abnormalities would have also disappeared. It was felt that the results would be affected less by having slightly different cohorts than by excluding new patients.

The drop in abnormal results relating to glucose from 2006 to 2007 may reflect the change in test used to screen for glucose. Glycated haemoglobin was used originally as it was assumed that patients could not fast reliably; however, when lipids were being monitored, patients had to fast anyway, and so this concern seemed groundless - one intervention put in place here was providing an information sheet with clear instructions on fasting and reminder phone calls or text messages. The halving of abnormal results over the 2 years and the doubling of detection of diabetes suggested that fasting glucose was a more meaningful test than $\mathrm{HBA}_{1 \mathrm{c}}$ in the clinic setting. Certainly, using $\mathrm{HBA}_{1 \mathrm{c}}$ as a screening tool at ten times the price per test compared with fasting glucose does not, on the basis of the results demonstrated here, seem viable. This introduced a bias in terms of follow-up because of the very small numbers of abnormal results detected in the second year. However, this result is a finding in itself in terms of improving healthcare and may add to the argument in support of specialist services for physical healthcare; as such, a service would be more likely to keep up to date with guideline developments and regulation and improvement of monitoring through audit. Glycated haemoglobin was still used for monitoring adherence to diabetic care in patients with diabetes.

Out-of-date results were included with abnormal results that were underinvestigated because out-of-date monitoring can have the same consequences in terms of patient safety as poor follow-up. Fasting lipid tests were the most frequent out-of-date results. In 2006 there was confusion around how to implement the tests properly. In 2007 the eight out-of-date tests were owing to patient non-adherence to fasting.

Fourteen patients were taking other antipsychotics and mood stabilisers. The inclusion of these patients might have increased overall prevalence of cardiovascular risk factors, and so the findings from the present study can only be related to patients in a clozapine clinic rather than to patients on clozapine more generally.

\section{Further work}

Clinically, although not formally assessed, no critical incidents have occurred since the intervention. In the time since the intervention, the diagnosis and subsequent treatment of diabetes have almost doubled, and $20 \%$ of patients within the clinic now have appropriate treatment and follow-up for high cholesterol, triglycerides, hypertension and diabetes when previously they were not on treatment. The implementation of guidelines for follow-up has had a significant impact on this, as having a smaller group of properly triaged patients sent to the GP for follow-up means resources can be concentrated in supporting those few patients in accessing primary care or secondary care medical services. This has improved primary care follow-up substantially in a cost-effective way. Most patients (44 of 51 by the end of the trial period) have a cardiovascular risk profile and intervention plan in their psychiatric notes that has been discussed with them and communicated to the GP and care coordinator before the intervention; out of 51 sets of notes, only 2 had communication from the GP relating to the annual health check. Many patients have been appreciative of this, 'taken ownership' of their problems and been motivated to make changes. This may indicate a need for more investment in specialist services for patients who require physical health monitoring in secondary care, but the most pertinent evidence to support investment comes from the data relating to the high prevalence of cardiovascular risk factors. Further investment would be used to train nurses to deliver interventions on addressing obesity, smoking and health education using training courses run by the public health department, to provide resources for such interventions, and to fund further research into the short- and long-term efficacy of such approaches in reducing cardiovascular risk factors in this population. Training mental health nurses to become specialists in physical health is important, as dual training is key to providing members of staff who can effectively bridge the physical/mental divide, coordinate physical healthcare, and provide education about physical health conditions and interventions to the community mental health teams. This is important as NHS mental health trusts are increasingly being asked to provide evidence of physical health provision for their patients. One further result was that by designing a new service model, the old model was streamlined and updated, guidelines and procedures were put in place, and standards for auditing purposes were developed. As a result, the qualified nurse sessions for the clinic were reduced from ten to six sessions - a cost saving. The clinic did, however, gain a session of occupational therapy time to carry out healthy lifestyle interventions and assessments. The pharmacist for the clinic also trained to become an independent prescriber and sees clients for cardiovascular risk assessment.

It would also be useful to look at the effect of these approaches on mental health. An observation from the clinic was that for some of the patients engaging with these measures, a significant improvement in mood and a reduction in some of the negative symptoms of schizophrenia were observed. Although it could be argued that such patients could attend mainstream services for these interventions, in reality it is increasingly recognised (by the 
number of specialist services that are starting to be developed) that this is ineffective as the core negative symptoms of schizophrenia - apathy and amotivation combined with mild cognitive impairment and blunted affect - often mean that a very different approach, with specialist understanding of these difficulties, is required. The nurses also had training in motivational interviewing to assist with this, provided by the drug and alcohol service. Patient education about medication, how schizophrenia affects health and dual diagnosis issues were addressed in 'healthy lifestyle groups' run by the nurses, which would not be available in mainstream services. Furthermore, many of the patients had had negative experiences of primary care interventions to reduce cardiovascular risk - a finding that is consistent with the literature. ${ }^{18}$ One proposed way (yet to be implemented) of bridging the gap between mainstream and specialist services was a pilot whereby the public health department provides a lifestyle trainer who works within the clinic to help provide one-to-one interventions with this population. This was part of a scheme set up for population groups with health challenges. Further investment could potentially be sourced from a 'shared care' model, whereby GPs share a proportion of their 'severe mental illness' payments with the clinic in return for the clinic conducting the annual health check that GPs require to receive the payment, with the understanding that GPs then provide the follow-up, with communication with secondary care services.

In summary, specialist secondary care clinics are important in providing improved follow-up and coordinated physical healthcare for patients on clozapine, and further investment in such services should be considered.

\section{About the author}

Philippa J. Bolton is senior registrar in adult psychiatry, Early Intervention in Psychosis Service, Bishop Auckland, UK.

\section{References}

1 Newman SC, Bland RC. Mortality in a cohort of patients with schizophrenia: a record linkage study. Can J Psychiatry 1991; 36: 239-45.

2 Nasrallah HA, Meyer JM, Goff DC, McEvoy JP, Davis SM, Stroup TS, et al. Low rates of treatment for hypertension, dyslipidemia and diabetes in schizophrenia: data from the CATIE schizophrenia trial sample at baseline. Schizophr Res 2006; 86: 15-22.
3 National Institute for Health and Clinical Excellence. Guidelines for Schizophrenia (Clinical Guideline 82). NICE, 2006 (http:// guidance.nice.org.uk/CG82/NiceGuidance/pdf/English).

4 Barnes TRE, Paton C, Cavanagh MR, Hancock E, Taylor D. A UK audit of screening of metabolic side effects of antipsychotics in community patients. Schizophr Bull 2007; 33: 1397-403.

5 Fontaine $K R$, Heo $M$, Harrigan EP, Shear CL, Lakshminarayanan $M$, Casey DE, et al. Estimating the consequences of antipsychotic induced weight gain on health and mortality rates. Psychiatry Res 2001; 101: 277-88.

6 Taylor D, Paton C, Kerwin R. The Maudsely 2005-2006 Prescribing Guidelines, 8th edn. Taylor \& Francis, 2005.

7 Which atypical antipsychotic drug for schizophrenia? Drug Ther Bull 2004; 42: 57-60.

8 National Institute for Health and Clinical Excellence. Hypertension: Management of Hypertension in Adults in Primary Care (Clinical Guideline 34). NICE, 2006 (http://www.nice.org.uk/nicemedia/pdf/CG034NICE guideline.pdf)

9 National Institute for Health and Clinical Excellence. Type 1 Diabetes: Diagnosis and Management of Type 1 Diabetes in Children, Young People and Adults (Clinical Guideline 15). NICE, 2004 (http://www.nice.org.uk/ nicemedia/pdf/CG015NICEguideline.pdf)

10 Smellie WSA. Testing pitfalls and summary of guidance in lipid management. BMJ 2006; 333: 83-6.

11 Smellie WSA, Forth J, Bareford D. Best practice in primary care pathology: review 3. J Clin Pathol 2006; 59: 1116.

12 Smellie WSA, Forth J, Ryder S, Galloway MJ, Wood AC, Watson ID. Best practice in primary care pathology: review 5. J Clin Pathol 2006; 59: 1229-37.

13 Scanlan M, Houltram B. A Care Map for the Assessment and Management of Atypical Antipsychotic Side Effects. University College, Northampton, 2006.

14 Henderson DC, Caligero E, Gray C. Clozapine, diabetes mellitus, weight gain, and lipid abnormalities: a five-year naturalistic study. Am J Psychiatry 2000; 157: 975-81.

15 Henderson DC, Daley TB, Kunkel L, Rodrigues-Scott M, Koul P, Hayden D. Clozapine and hypertension: a chart review of 82 patients. J Clin Psychiatry 2004; 65: 686-9.

16 Lamberti JS, Costea GO, Olson D, Crilly JF, Maharaj K, Tu X, et al. Diabetes mellitus among outpatients receiving clozapine: prevalence and clinical - demographic correlates. J Clin Psychiatry 2005; 66: 900906.

17 Lamberti J, Olson D, Crilly J, Olivares T, Williams GC, Tu X, et al. Prevalence of metabolic syndrome amongst patients receiving clozapine. Am J Psychiatry 2006; 163: 1273-6.

18 Ucok A, Soygur H, Atakli C, Kuscu K, Sartorius N, Duman ZC, et al. The impact of antistigma education on the attitudes of general practitioners regarding schizophrenia. Psychiatry Clin Neurosci 2006; 60: 439-43. 